\title{
Specific localization of nesprin-1-a2, the short isoform of nesprin-1 with a KASH domain, in developing, fetal and regenerating muscle, using a new monoclonal antibody
}

lan Holt ${ }^{1,2^{*}}$, Nguyen Thuy Duong ${ }^{1,3}$, Qiuping Zhang ${ }^{4}$, Le Thanh Lam ${ }^{1}$, Caroline A. Sewry ${ }^{1,5}$, Kamel Mamchaoui ${ }^{6}$, Catherine M. Shanahan ${ }^{4}$ and Glenn E. Morris ${ }^{1,2}$

\begin{abstract}
Background: Nesprin-1-giant (1008kD) is a protein of the outer nuclear membrane that links nuclei to the actin cytoskeleton via amino-terminal calponin homology domains. The short nesprin-1 isoform, nesprin-1-a2, is present only in skeletal and cardiac muscle and several pathogenic mutations occur within it, but the functions of this short isoform without calponin homology domains are unclear. The aim of this study was to determine mRNA levels and protein localization of nesprin-1-a2 at different stages of muscle development in order to shed light on its functions.

Results: mRNA levels of all known nesprin-1 isoforms with a KASH domain were determined by quantitative PCR. The mRNA for the 111 kD muscle-specific short isoform, nesprin-1-a2, was not detected in pre-differentiation human myoblasts but was present at significant levels in multinucleate myotubes. We developed a monoclonal antibody against the unique amino-terminal sequence of nesprin-1-a2, enabling specific immunolocalization for the first time. Nesprin-1-a2 protein was undetectable in pre-differentiation myoblasts but appeared at the nuclear rim in post-mitotic, multinucleate myotubes and reached its highest levels in fetal muscle. In muscle from a Duchenne muscular dystrophy biopsy, nesprin-1-a2 protein was detected mainly in regenerating fibres expressing neonatal myosin. Nesprin-1-giant was present at all developmental stages, but was also highest in fetal and regenerating fibres. In fetal muscle, both isoforms were present in the cytoplasm, as well as at the nuclear rim. A pathogenic early stop codon (E7854X) in nesprin-1 caused reduced mRNA levels and loss of protein levels of both nesprin-1-giant and (unexpectedly) nesprin-1-a2, but did not affect myogenesis in vitro.
\end{abstract}

Conclusions: Nesprin-1-a2 mRNA and protein expression is switched on during myogenesis, alongside other known markers of muscle differentiation. The results show that nesprin-1-a2 is dynamically controlled and may be involved in some process occurring during early myofibre formation, such as re-positioning of nuclei.

Keywords: SYNE1, Nuclear membrane, Monoclonal antibody, Cardiomyopathy, Emery-Dreifuss muscular dystrophy, Lamin A/C

\footnotetext{
* Correspondence: ian.holt@rjah.nhs.uk

${ }^{1}$ Wolfson Centre for Inherited Neuromuscular Disease, RJAH Orthopaedic

Hospital, Oswestry, SY10 7AG, UK

${ }^{2}$ Institute for Science and Technology in Medicine, Keele University, Keele

ST5 5BG, UK

Full list of author information is available at the end of the article
} 


\section{Background}

Nuclear envelope spectrin-repeat proteins (nesprins) form a structural link between the nuclear envelope and cytoskeletal filaments. The SYNE1 gene, which encodes nesprin-1, was identified by yeast two-hybrid screening of fetal mouse post-synaptic membrane cDNA [1] and by differential screening of a rat vascular smooth muscle cell cDNA [2]. These early studies also identified a related gene, SYNE2, which encodes nesprin-2 [1, 2]. The protein products originally identified were, in fact, shorter C-terminal isoforms of larger proteins, nesprin1 -giant $(1008 \mathrm{kD})$ and nesprin-2-giant (792kD) [2-4]. The SYNE1 gene on human chromosome $6 \mathrm{q} 25$ is also known as MYNE1 [5] or Enaptin [6] and the SYNE2 gene on human chromosome $14 \mathrm{q} 23$ is also known as NUANCE [3]. Both giant nesprins have calponin homology $(\mathrm{CH})$ domains at their N-terminals that bind the actin cytoskeleton, and transmembrane Klarsicht-ANCSyne-homology (KASH) domains at their C-terminals that bind to inner nuclear membrane SUN proteins in the luminal gap between inner and outer nuclear membranes [7, 8]. Additionally, on the nucleoplasmic side of the inner nuclear membrane, SUN proteins interact with A-type lamin components of the nuclear lamina. These linker of nucleoskeleton and cytoskeleton (LINC) complexes form a physical connection joining the cytoskeleton and the nucleus $[9,10]$. Several human mutations in the $\mathrm{C}$-terminal region of nesprin-1 are associated with Emery-Dreifuss muscular dystrophy (EDMD) and dilated cardiomyopathy [11-14]. Mutations in nesprininteraction partners, emerin (EMD, [15] and lamin $\mathrm{A} / \mathrm{C}$ (LMNA, [16]) account for about $50 \%$ of EDMD cases (reviewed [17]).

SYNE1 and SYNE2 have multiple internal promoters which may give rise to shorter nesprin isoforms which are truncated at the $\mathrm{N}$-terminus but have a common C-terminal region. Three additional members of the nesprin family (nesprin-3, nesprin-4 and KASH5) are similar to the shorter nesprin isoforms in that they lack N-terminal CH domains. Nesprin-3 (112kD) contains a plectin-binding domain at the $\mathrm{N}$-terminal which interacts with intermediate filaments [18], nesprin-4 (44kD) interacts with microtubules via kif5b [19] and KASH5 (63kD) links to chromosomes via dynein $[20,21]$. These functional products of 3 separate, but related, genes suggest possible related functions for the similar short products of the $S Y N E 1 / 2$ genes.

We recently showed by qPCR that mRNAs for nesprin short forms were present at only very low levels in most of the 20 human tissues studied, but were significantly expressed in specific cell types or stages of development [22]. Thus, nesprin-1- $\alpha 2$ was found in skeletal and cardiac muscle only and nesprin-2-epsilon-1 was associated with cells at very early stages of development, while nesprin-2-epsilon-2 was expressed in cardiac, but not skeletal, muscle [22].

In the present study, we extended our qPCR studies of adult human tissues to different stages of skeletal muscle development and, finding that the mRNA for nesprin-1$\alpha 2$ appeared only after myogenic differentiation, we developed a new monoclonal antibody specific for the unique N-terminal amino-acid sequence of nesprin-1- $\alpha 2$ for immunolocalization studies.

\section{Results}

We have studied four stages of human muscle development: pre-differentiation, dividing myoblast cultures, differentiated myotube cultures expressing muscle-specific proteins, fetal muscle and adult muscle. We have also studied dystrophic muscle containing a significant proportion of immature fibres and muscle cultures with pathogenic mutations in nesprin-1 and lamin $\mathrm{A} / \mathrm{C}$.

\section{Nesprin-1-a2 mRNA appears during myogenesis in vitro and nesprin-1-giant mRNA increases}

mRNA levels in control human cells were determined by quantitative PCR (qPCR: Table 1) with appropriate standards and controls (see Methods). As expected, mRNA for the muscle differentiation marker, M-creatine kinase (CKM), was absent from dividing myoblasts, but increased markedly during muscle development (Fig. 1b). mRNA for the muscle cell marker protein, desmin, also increased; it was already present in the committed, though still dividing myogenic cells, as expected (Fig. 1b). Like CKM, nesprin-1- $\alpha 2$ mRNA was absent from myoblasts, but present in myotubes (Fig. 1a). Nesprin-1giant mRNA was present in myoblasts, but also higher in myotubes (Fig. 1a). Other short isoforms of nesprin-1 did not make a major contribution to total nesprin mRNA content of cultured cells (Table 1). For these control myogenesis studies in vitro, five different transformed myoblast lines were used (Table 1), derived from control subjects of different ages and each individual cell line showed changes comparable to the mean values shown in Fig. 1a.

\section{Effects of pathogenic mutations in nesprin-1 and lamin A/C on mRNA levels of nesprin-1 isoforms during myogenesis}

Myoblast cell lines were also derived from a muscular dystrophy patient with an early termination mutation in the nesprin-1 gene [23], located just before the start of the short nesprin-1- $\alpha 2$ isoform. One would expect all nesprin-1 mRNAs to be synthesised normally, though nesprin-1-giant mRNA might be less stable than normal, because of "nonsense-mediated decay". In fact, consistent with reduced mRNA stability, the level of nesprin-1giant mRNA was reduced by $78 \%$ in both myoblasts and myotubes compared to the means of the 5 control 


\begin{tabular}{|c|c|c|c|c|c|c|c|c|c|c|}
\hline & \multicolumn{2}{|l|}{ Control 5 days } & \multicolumn{2}{|c|}{ Control 25 years } & \multicolumn{2}{|l|}{ Control 41 years } & \multicolumn{2}{|l|}{ Control 53 years } & \multicolumn{2}{|l|}{ Control 79 years } \\
\hline & Myoblast & Myotube & Myoblast & Myotube & Myoblast & Myotube & Myoblast & Myotube & Myoblast & Myotube \\
\hline \multirow[t]{2}{*}{ N1-Giant } & $61.1 \pm 11.8(5)$ & $130.0 \pm 12.8(6)$ & $88.3 \pm 12.3(6)$ & $206.3 \pm 17.3$ & $47.7 \pm 4.6(4)$ & $113.7 \pm 19.3$ & $47.4 \pm 6.7(5)$ & $172.4 \pm 22.1$ & $60.5 \pm 10.1$ & $212.4 \pm 12.1$ \\
\hline & $P<0.001$ & & $P<0.001$ & & $P<0.001$ & & $P<0.001$ & & $P<0.001$ & \\
\hline$N 1-\beta 1$ & $<1(1)$ & $<1(1)$ & $<1(2)$ & $<1(2)$ & $<1(1)$ & $<1(1)$ & $<1(1)$ & $<1(1)$ & $1.1(1)$ & $1.2(1)$ \\
\hline$N 1-\beta 2$ & $<1(1)$ & $1.2(1)$ & $<1(3)$ & $2.1 \pm 0.8(3)$ & $<1(1)$ & $2.7(1)$ & $<1(1)$ & $<1(1)$ & $<1(1)$ & $1.2(1)$ \\
\hline N1-a1 & nd (1) & nd (1) & nd (1) & nd (1) & nd (1) & nd (1) & nd (1) & nd (1) & nd (1) & nd (1) \\
\hline \multirow[t]{2}{*}{$\mathrm{N} 1-\mathrm{a} 2$} & $<1(5)$ & $71.0 \pm 9.5(4)$ & $<1(3)$ & $47.5 \pm 9.3(4)$ & $<1(5)$ & $22.0 \pm 1.3(5)$ & $<1(5)$ & $44.7 \pm 9.1(4)$ & $<1(3)$ & $47.4 \pm 4.3(5)$ \\
\hline & $P<0.001$ & & $P<0.001$ & & $P<0.001$ & & $P<0.001$ & & $P<0.001$ & \\
\hline \multirow[t]{2}{*}{ CKM } & $<1(3)$ & $1158 \pm 116$ (3) & $<1(3)$ & $2847 \pm 285$ & $<1(3)$ & $740 \pm 186(3)$ & $<1(3)$ & $1884 \pm 104$ & $2.1 \pm 1.7$ (3) & $1362 \pm 68$ (3) \\
\hline & $P<0.001$ & & $P<0.001$ & & $P<0.005$ & & $P<0.001$ & & $P<0.001$ & \\
\hline \multirow[t]{2}{*}{ Desmin } & $2773 \pm 174$ (3) & $16434 \pm 157$ (3) & $6931 \pm 471$ & $16966 \pm 710$ & $3784 \pm 459$ & $7103 \pm 692$ (3) & $6532 \pm 605$ & $14799 \pm 798$ & $2484 \pm 478$ & $21973 \pm 1803$ \\
\hline & $P<0.001$ & & $P<0.001$ & & $P<0.005$ & & $P<0.001$ & & $P<0.001$ & \\
\hline
\end{tabular}

Results expressed as: Mean \pm SD (n), " $n$ " = number of repeat measurements. $n d=$ Not detected 


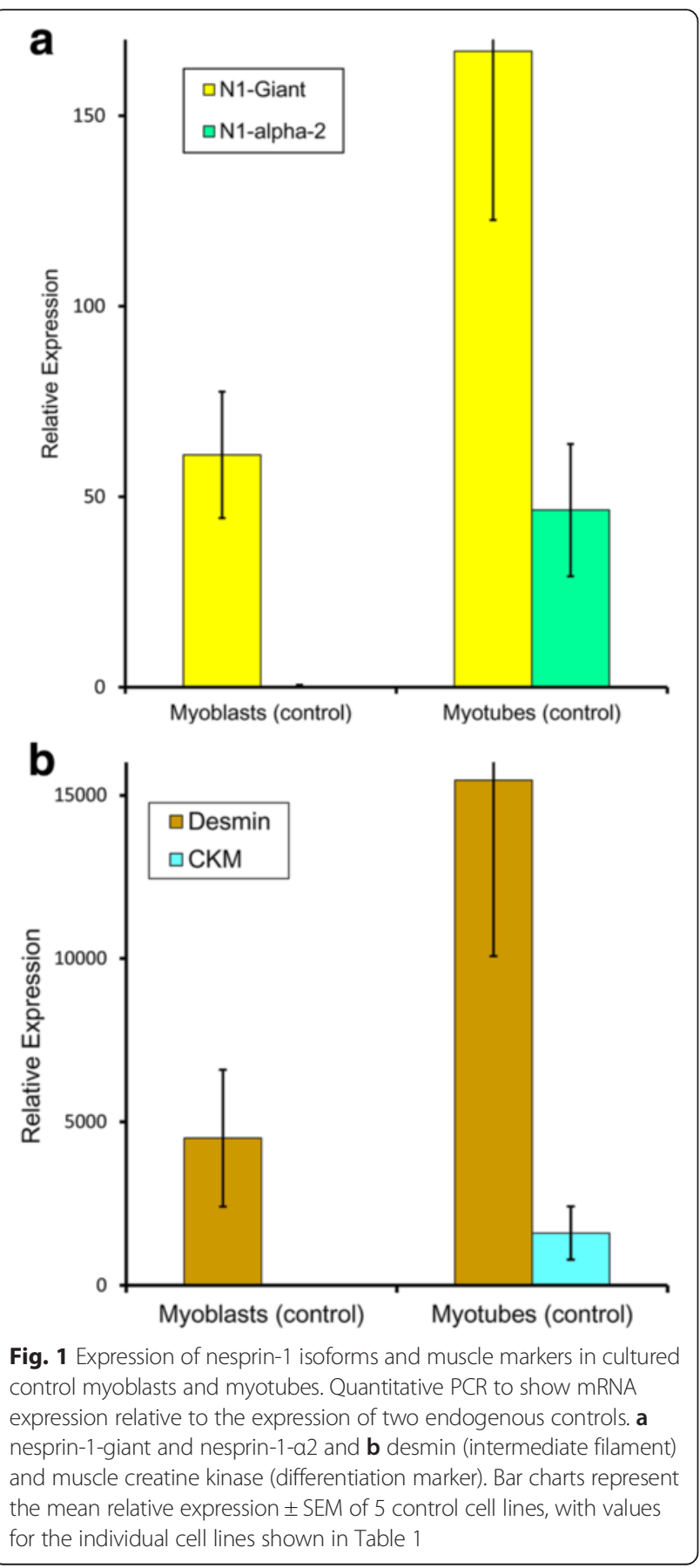

cell lines and was lower than the corresponding values in each of the 5 controls individually (Table 2). Surprisingly, the mRNA for nesprin-1- $\alpha 2$ was also reduced by $85 \%$ in the mutant myotubes, even though the mutation lies 5' to its first exon. Levels of muscle marker mRNAs for CKM and desmin were unaffected by this mutation. Nesprins at the outer nuclear membrane are linked to lamin $\mathrm{A} / \mathrm{C}$ in the nucleus via SUN proteins in the lumen of the nuclear membrane [10]. Therefore, we also studied myoblast cell lines with mutations in the
LMNA gene, which encodes lamin $\mathrm{A} / \mathrm{C}$. Among the 3 myoblast lines from patients with 3 different mutations in the $L M N A$ gene $[24,25]$, there was no consistent effect on the mRNA levels for nesprin-1 shown by all 3 LMNA mutant cell lines (Table 2). Nesprin-1-giant mRNA levels were very high in one LMNA mutant (L380S), but it is not clear, without the availability of additional similar cell lines, whether this is attributable to the specific mutation.

\section{New monoclonal antibodies against nesprin-1 isoforms}

Having established the changes in mRNA levels, it was necessary to determine whether protein levels changed in the same way. Since antibodies specific for short isoforms were not available, we produced new monoclonal antibodies and these are shown in Table 3. Human nesprin-1- $\alpha 2$ has a unique 31 aa sequence at its aminoterminus and a synthetic peptide corresponding to amino acids 2-17 was linked to KLH via its C-terminal cysteine. This peptide conjugate was used to produce a monoclonal antibody (mAb: N1alpha2-1H2) specific for this short isoform, as described in "Experimental Procedures". Another 16 amino acid peptide within exon 130 was used similarly to produce a second mAb, N1GEx130, which does not recognise nesprin-1- $\alpha 2$, but it does recognise nesprin-1-giant and would recognise beta isoforms. Beta isoforms are only expressed at low levels in skeletal muscle relative to the giant form (Table 3 and [22]), but to exclude them we also produced mAbs corresponding to exons 81-86 of the nesprin-1 gene (Table 3). These mAbs will recognise nesprin-1-giant but not the beta or alpha isoforms. The epitope locations of the new nesprin-1 mAbs, along with the MANNES1A and MANNES1E C-terminal mAbs (Randles et al., 2010 [26]), are illustrated in Fig. 2. All of the exon 81-86 mAbs recognised a band of nesprin-1-giant on western blots of a human skin fibroblast extract (Fig. 3a), the same band as recognised by a previously-established mAb, MANNES1E [26]. On western blot of human skeletal muscle extract, mAb N1G-Ex130, like MANNES1E, gave a band of nesprin-1-giant (Fig. 3b). For verification of the size of the nesprin-1-giant band, human skeletal muscle extract was run on a gradient gel along with a molecular weight marker with an upper band of 250 $\mathrm{kDa}$ (Fig. 3c). As an additional size marker, $\mathrm{mAb}$ MANDRA1 was used to show a band of dystrophin protein at $427 \mathrm{kDa}$. Nesprin-1 mAbs recognise bands that are larger than $427 \mathrm{kDa}$ and are consistent with nesprin-1-giant at around $1008 \mathrm{kDa}$ (Fig. 3c).

The mAb N1G-Ex130 and the N1G-Exon 81-86 mAbs against the giant isoform recognise nesprin-1-giant, but would not be expected to recognise the short $\alpha 2$ isoform, on a western blot of adult human muscle, whereas MANNES1E does recognise a band of the size expected for 
Table 2 Relative expression of mRNA of nesprin-1 isoforms and markers of differentiation in cultured human myoblasts and myotubes from patients with pathogenic mutations in Syne1 or LMNA

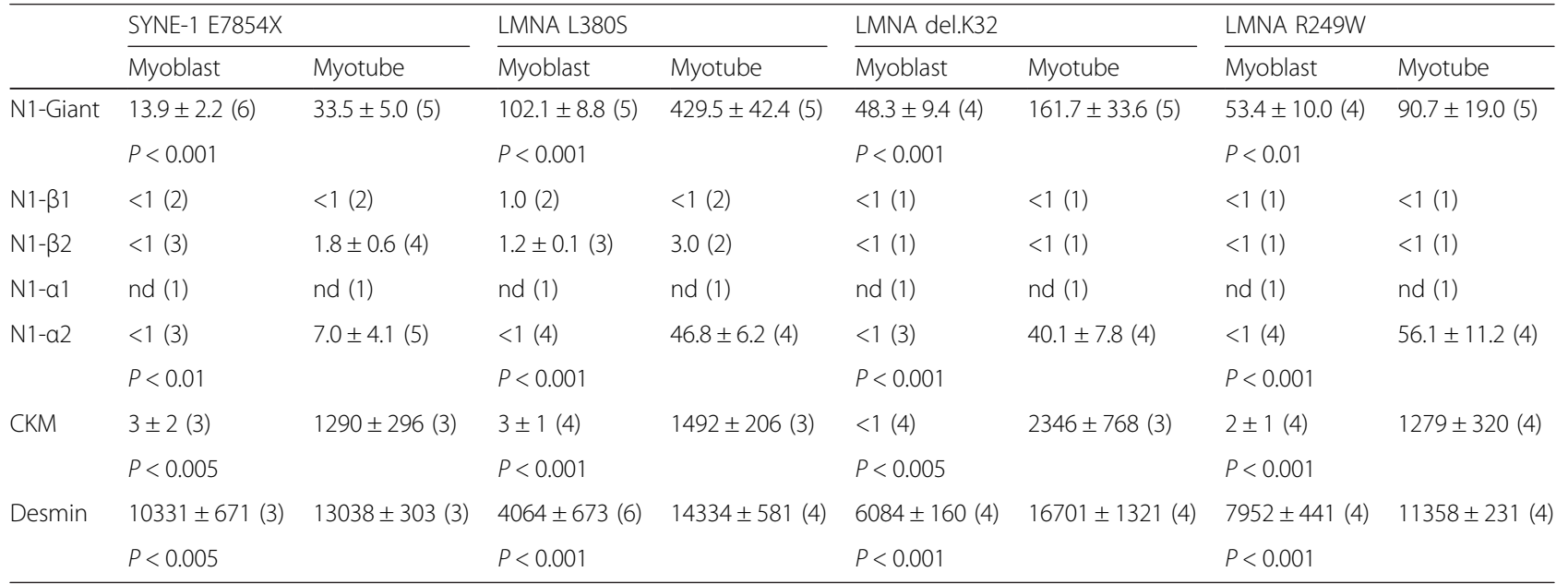

Results expressed as: Mean \pm SD $(n), " n$ " = number of repeat measurements. $n d=$ Not detected

nesprin-1- $\alpha 2$ (Fig. $3 \mathrm{~b}$ and c). We cannot confirm that this band is nesprin-1- $\alpha 2$, because the $\alpha 2$-specific $m A b$, N1alpha2-1H2, does not work on western blots, but the specificity of the mAb is suggested by the absence of nuclear rim staining both in cells that do not express nesprin$1-\alpha 2$, such as skin fibroblasts (not shown), and in myotubes with $85 \%$ reduced $\alpha 2$ mRNA levels (SYNE1 mutant cell line: Fig. 4b). The advantage of using N1G-Ex130 to detect nesprin-1-giant, instead of the exon $81-86 \mathrm{mAbs}$, is that the exon 130 region does not encode any known $\mathrm{N}$-terminal (KASH-less) isoforms of nesprin-1, whereas such isoforms containing the exon 81-86 region have been detected at the mRNA level [27]. There may be very few nesprin-1 sequences that are unique to the giant isoform.

Table 3 Nesprin-1 monoclonal antibodies used in this study

\begin{tabular}{|c|c|c|c|c|}
\hline mAb name & Isotype & Epitope & Western blot & IMF \\
\hline & & $\begin{array}{l}\text { Unique sequence preceding } \\
\text { Exon } 131 \text { of N1-Giant }\end{array}$ & & \\
\hline \multirow[t]{2}{*}{ N1alpha2-1H2 } & $\operatorname{lgG} 1$ & $\begin{array}{l}\text { WAEDLSALRMAEDGC (aa 2-17 } \\
\text { of unique N1-alpha-2 sequence) }\end{array}$ & - & $\begin{array}{l}++ \text { (myotubes, immature and } \\
\text { regenerating fibres) }\end{array}$ \\
\hline & & Exon 130 & & \\
\hline \multirow[t]{2}{*}{ N1G-Ex130 } & $\lg \mathrm{lg} 1$ & SKASEIEYKLGKVNDRC & +++ & +++ \\
\hline & & Exons 81-84 & & \\
\hline N1G-7D9 & $\lg \mathrm{lg} 1$ & QDKLPGSSA (Exon 82) & +++ & + \\
\hline N1G-8C8 & $\operatorname{lgG} 1$ & - & ++ & + \\
\hline N1G-6F7 & $\lg \mathrm{lg} 1$ & - & + & + \\
\hline \multirow[t]{2}{*}{$\mathrm{N} 1 \mathrm{G}-4 \mathrm{C} 11$} & $\lg G 2 b$ & EMIDQLQDKLP (Exon 82) & $+($ cross reaction $)$ & + (cross reaction) \\
\hline & & Exons 84-86 & & \\
\hline N1G-7C8 & $\operatorname{lgG} 1$ & - & ++ & ++ \\
\hline $\mathrm{N} 1 \mathrm{G}-5 \mathrm{~A} 6$ & $\lg G 1$ & - & ++ & + \\
\hline N1G-9G5 & $\lg \mathrm{g} 1$ & LGLYTILPSELSL (Exon 84) & +++ & + \\
\hline \multirow[t]{2}{*}{ N1G-6C9 } & $\operatorname{lgG} 1$ & LKIRDQIQDK (Exon 84-85) & $+($ cross reaction $)$ & + \\
\hline & & Exons143-146 & & \\
\hline MANNES1A & $\operatorname{lgG} 1$ & - & ++ & ++++ \\
\hline MANNES1E [25] & $\lg \mathrm{lg} 1$ & - & +++ & +++ \\
\hline
\end{tabular}

Except for MANNES1A and MANNES1E (Randles et al., 2010 [26]), all other Nesprin-1 mAbs in the table are reported here for the first time Exon numbering is based on the SYNE1 transcript variant 1 mRNA (accession: NM_182961.3) and is the same as that used by Rajgor et al., 2012 [27] 


- Isoform-specific region of Nesprin-1 $\alpha 2$ 2
Fig. 2 Pictorial representation of nesprin-1-giant and nesprin-1-a2 proteins with locations of monoclonal antibody (mAb) epitopes. Nesprin-1-a2
has a unique N-terminal sequence, but is otherwise identical to the C-terminal region of Nesprin-1-giant, including the unstructured and highly
conserved "Star" region [28] and the alternatively spliced and highly conserved "DV23" exon [1, 4, 22, 28]. The N-terminal start point of nesprin-1-
beta-1 relative to nesprin-1-giant, is shown as a black arrow. Red arrows indicate positions of the epitopes of mAbs against nesprin-1 that
have been used in this study. Monoclonal antibodies MANNES1A and MANNES1E recognise the common C-terminal region of nesprin-1-giant and
nesprin-1-a2 [26]. The new mAb, N1alpha2-1H2 recognises the unique N-terminal region of nesprin-1-a2 and the other new mAbs recognise nesprin-
1-giant but not nesprin-1-a2

Nesprin-1-a2 protein is located mainly at the nuclear rim in myotube nuclei and is absent from myoblast nuclei N1alpha2-1H2 mAb does not stain myoblasts, but does stain the nuclear rim in control myotubes (Fig. 4a), consistent with mRNA data showing the first appearance of nesprin-1- $\alpha 2$ mRNA at the myotube stage (Fig. 1). Pre-incubation of N1alpha2-1H2 with the peptide epitope used for immunization abolished immunostaining of myotubes, whereas control pre-incubation with an unrelated peptide did not (Fig. 5). In contrast to N1alpha2-1H2, MANNES1A (against both giant and alpha isoforms) stains the nuclear rim both in dividing myoblasts and in post-mitotic multinucleate myotubes (Fig. 4c). There is also some cytoplasmic staining in myotubes with N1alpha2-1H2 (Fig. 4a) and to a lesser extent, with MANNES1A (Fig. 4c). Nesprin-1-giant-specific mAbs at both exon 130 (N1GEx130, Fig. 4a) and exons 84-86 (N1G-7C8, Fig. 4c) confirm that nesprin-1-giant is present at the nuclear rim throughout myogenesis in vitro.

Myotube cultures of the early termination nesprin-1 mutant were negative for all nesprin- $1 \mathrm{mAbs}$, including the N1alpha2-1H2 mAb (Fig. 4b). This may seem surprising since the stop codon is 5' to the nesprin-1- $\alpha 2$ sequence, but qPCR shows that nesprin-1- $\alpha 2$ mRNA levels are only $15 \%$ of control levels (Tables 1 and 2 ) and this may result in protein levels below the level of detection. The more surprising observation, therefore, is that the nesprin-1- $\alpha 2$ mRNA is so much reduced. There is the possibility of a truncated form of nesprin-1-giant protein, but qPCR (Tables 1 and 2) shows that this would probably exist at only 20-25\% of normal levels, even assuming it were as stable as the full-length protein. In fact, neither N1G-Ex130 (Fig. 4b) nor the nesprin-1specific exon 81-86 mAbs (not shown) detected any nesprin-1-giant in the nesprin-1 mutant myotubes, confirming that truncated mutant protein was present at only very low levels, if at all.

Nesprin-1-a2 protein is weakly expressed in mature muscle fibres, but is strongly expressed, alongside nesprin-1-giant in both fetal muscle and immature (regenerating) fibres of Duchenne muscular dystrophy muscle

Nuclei of human fetal skeletal muscle were clearly labelled with the N1alpha2-1H2 mAb (Fig. 6a) and low level cytoplasmic staining was also seen with this mAb. The large central nuclei are typical of immature muscle fibres, whereas mature muscle nuclei are compressed and peripheral. We were surprised to find that the N1alpha2-1H2 mAb only gave a weak, low level stain in many mature muscle fibres (Fig. 6b), suggesting that the short isoform is either present at low levels and/or the $\mathrm{mAb}$ has reduced accessibility to the epitope. Rarely $(<0.1 \%)$, normal human adult muscle nuclei were observed that were strongly positive for nesprin-1- $\alpha 2$ (not shown). Nesprin-1-giant was found at the nuclear envelope of both fetal (Fig. 6c) and adult (Fig. 6d) skeletal muscle, with additional low level cytoplasmic stain in fetal muscle. Figure 6 shows representative images of N1alpha2-1H2 and N1G-Ex130 immunofluorescent staining of a total of 3 fetal donors and 4 adult donors. Furthermore, when we studied a muscle section from a Duchenne muscular dystrophy patient, we found that regenerating fibres (identified by staining serial sections 

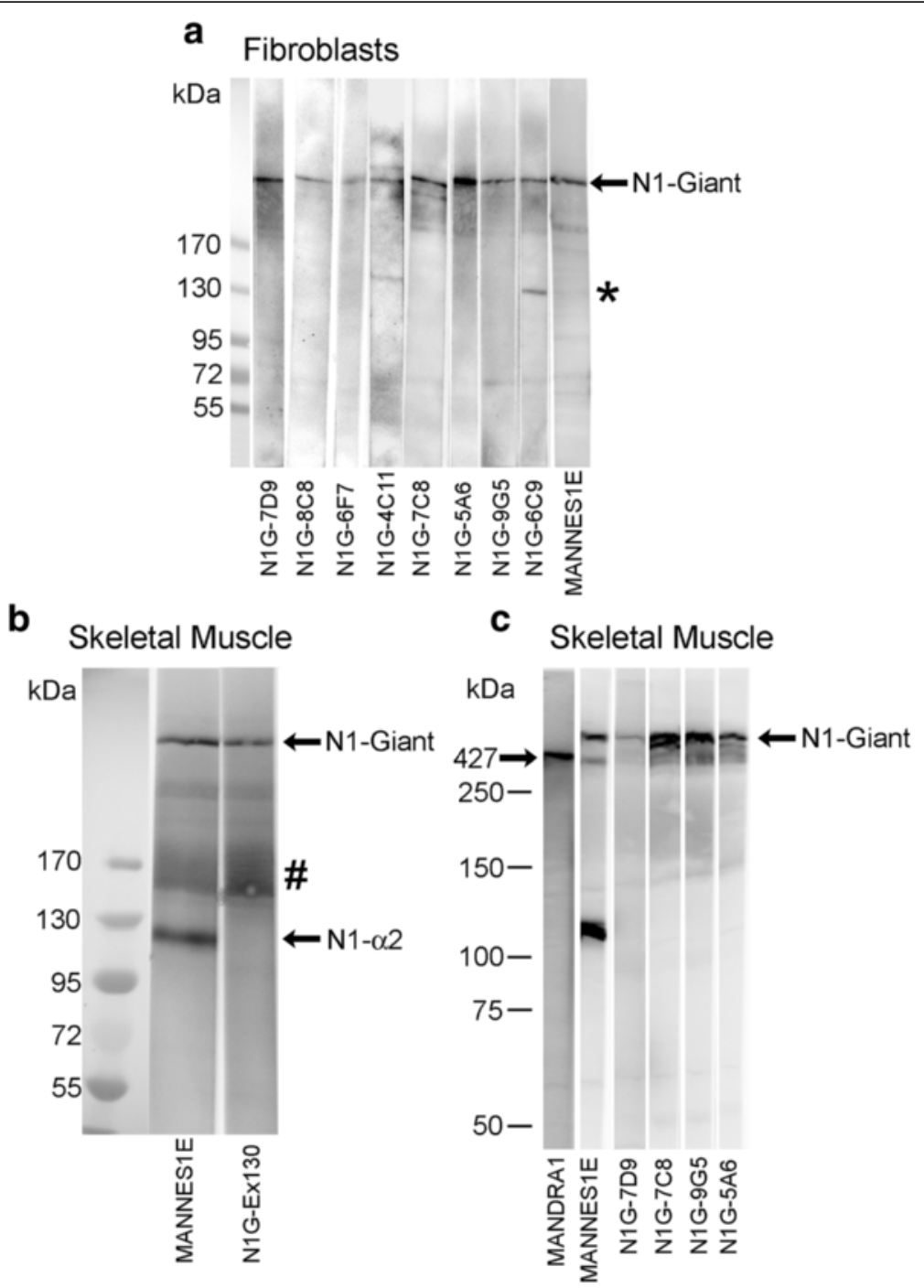

Fig. 3 Western blots with nesprin-1 mAbs. Extracts of a cultured human dermal fibroblasts probed with the new mAbs against exons 81 to 86 of nesprin-1-giant and $\mathbf{b}$ normal adult human skeletal muscle probed with the new mAb N1G-Ex130 against exon 130 of nesprin-1-giant. The molecular weight marker used for blots (a) and (b) was: EZ-Run Pre-Stained Rec Protein Ladder (Fisher; Cat No: BP3603), upper molecular weight 170 kDa. Blot (c) is human skeletal muscle extract alongside Precision Plus Protein Standards (BioRad; Cat No: 161-0374) as molecular weight markers, upper molecular weight $250 \mathrm{kDa}$. Strips of blot (c) were probed with MANDRA1 to show dystrophin as a size marker (427 kDa). Bands with nesprin-1 mAbs were higher than the $427 \mathrm{kDa}$ marker and consistent with nesprin-1-giant (1008 kDa). All blots include the C-terminal mAb against nesprin-1, MANNES1E [26]. * = likely non-specific bands; \# = likely nesprin degradation products

with neonatal myosin antibody specific for immature fibres) had high levels of nesprin-1- $\alpha 2$ protein (Fig. 7a). Although mainly at the nuclear rim, N1alpha2-1H2 mAb also showed some staining of the cytoplasm of regenerating fibres, with mature muscle fibres being much weaker (Fig. 7a). Using N1G-Ex130 mAb to locate nesprin-1giant, we found that this isoform was also higher in regenerating fibres, compared with mature fibres in the same section, and also showed faint cytoplasmic staining (Fig. 7b). Nesprin-1-giant was present at the nuclear rim of all nuclei in the muscle biopsy sections.

\section{Nesprin mRNA levels in fetal and adult human muscle} Both commercial and local sources were used for human fetal and adult skeletal muscle total RNAs, and total cDNAs were prepared by reverse transcription for qPCR quantification. Table 4 shows that nesprin isoform mRNA levels were highly variable between sources, although $\mathrm{M}$-creatine kinase and desmin mRNA levels were significantly higher in adult, compared with fetal, muscle, as expected. Although Fetal 1 and Adult 1 (from the same commercial source) in Table 4 showed changes in nesprin mRNA consistent 
a

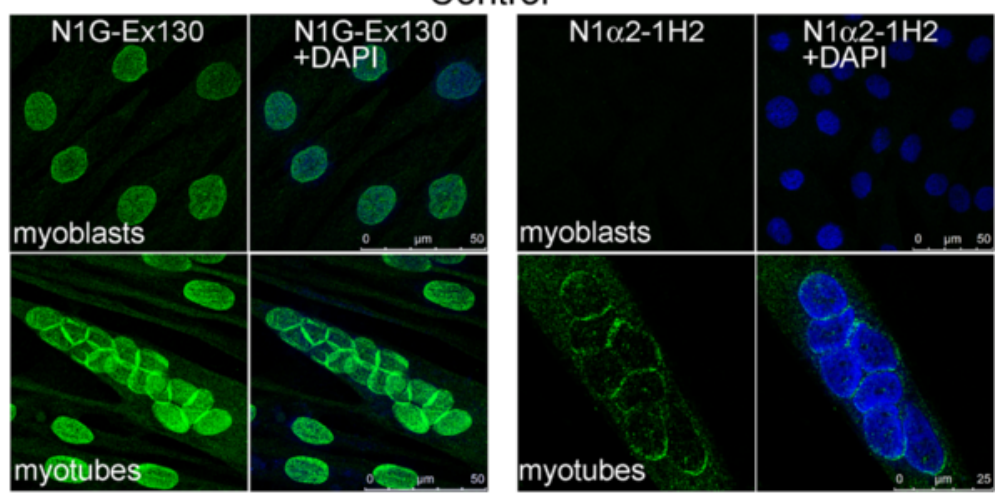

b

SYNE1 E7854X

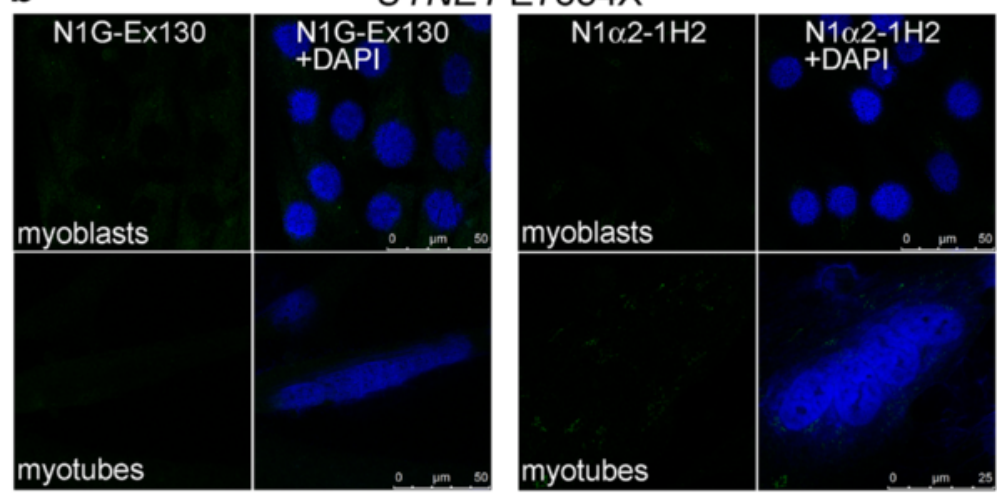

c

Control

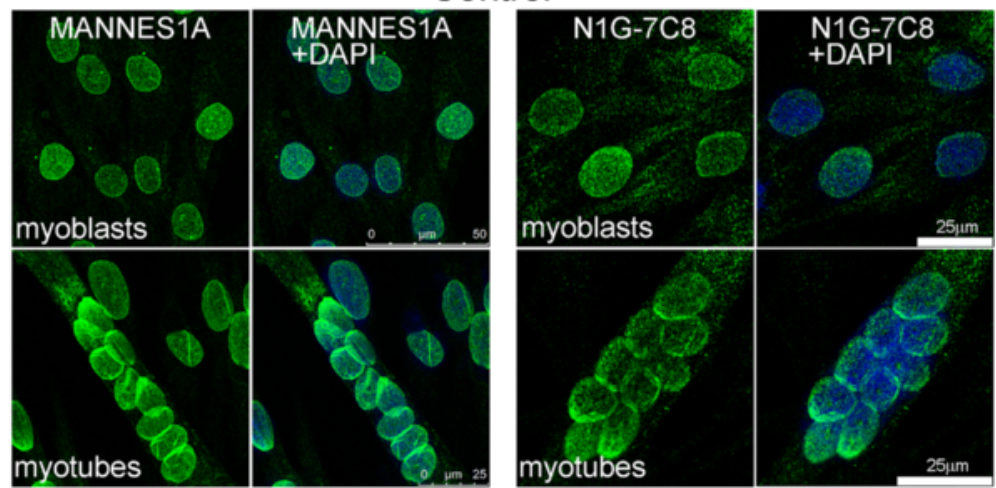

Fig. 4 Immunofluorescent staining for nesprin-1 in cultured myoblasts and myotubes. Individual microscopic fields are shown as paired images. The left image of each pair shows nesprin-1 antibody stain (green) and the right image of each pair shows the merged antibody (green) and DAPI (blue) nuclear stain. a mAb N1G-Ex130 stained the nuclei of control myoblasts and myotubes whereas mAb N1alpha2-1H2 stained the nuclear envelope of control myotubes but not myoblasts. $\mathbf{b}$ The mAbs did not stain nesprin-1 mutant myoblasts or myotubes. c Similar to the image seen with N1G-Ex130, mAb MANNES1A and also the mAbs targeted against exons 81-86 (N1G-7C8 shown), stained the nuclear rim of control myoblasts and myotubes

with the protein data (Figs. 4 and 6), they also expressed beta isoform mRNAs, not found in myotubes.

Human muscle tissue used for mRNA isolation will contain non-muscle cells, such as connective tissue, whereas the cultured cell lines are clonal and contain only desmin-positive myogenic cells. It is unclear, therefore, whether the beta isoform mRNAs in fetal and adult muscle are in myofibres (and therefore appear at some post-myotube stage) or in non-muscle components present in variable proportions in different muscle samples used for RNA extraction; this question can only be resolved with antibodies specific for the beta isoforms and these are not yet available. When we expanded our sampling to three fetal and five adult RNA preparations (Table 4), the results for all the nesprin-1 isoforms were much less consistent. It is possible that nesprin-1 mRNA 


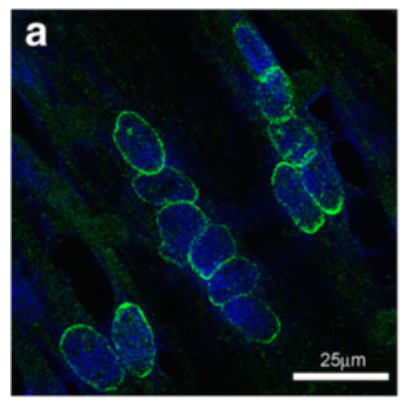

$\mathrm{N} 1 \alpha 2-1 \mathrm{H} 2$

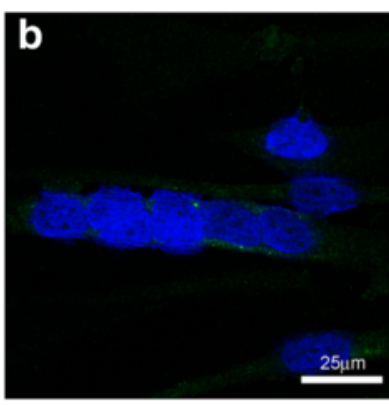

$\mathrm{N} 1 \alpha 2-1 \mathrm{H} 2$

$+\mathrm{N} 1 \alpha 2$ peptide

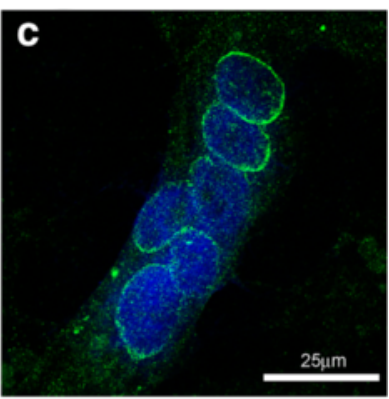

$\mathrm{N} 1 \alpha 2-1 \mathrm{H} 2$

+ unrelated peptide

Fig. 5 Peptide competition experiment. Localization of mAb N1alpha2-1H2 to the nuclear envelope of cultured myotubes (a) was neutralized when the mAb was blocked by pre-incubation with the immunizing peptide (b). Pre-incubation with an unrelated peptide did not prevent normal localization (c)

content is variably affected by tissue storage and methods of RNA extraction, since the qPCRs themselves were quite reproducible and gave good dissociation curves. Nesprin-1- $\alpha 2$ mRNA is barely detectable in dividing myoblasts, but accounts for up to one-third of all the nesprin-1 mRNA in adult muscle (Table 4), a proportion consistent with the western blot in Fig. $3 \mathrm{~b}$ and c.

\section{Discussion}

N1alpha2-1H2 is the first anti-nesprin-1 antibody that is specific for a single isoform since it recognises a sequence encoded by the unique first exon of nesprin-1$\alpha 2$. Antibodies against the $\mathrm{N}$-terminal region of nesprin1-giant will not recognize any other $\mathrm{KASH}+$ isoforms but a number of shorter $\mathrm{N}$-terminal isoforms are now
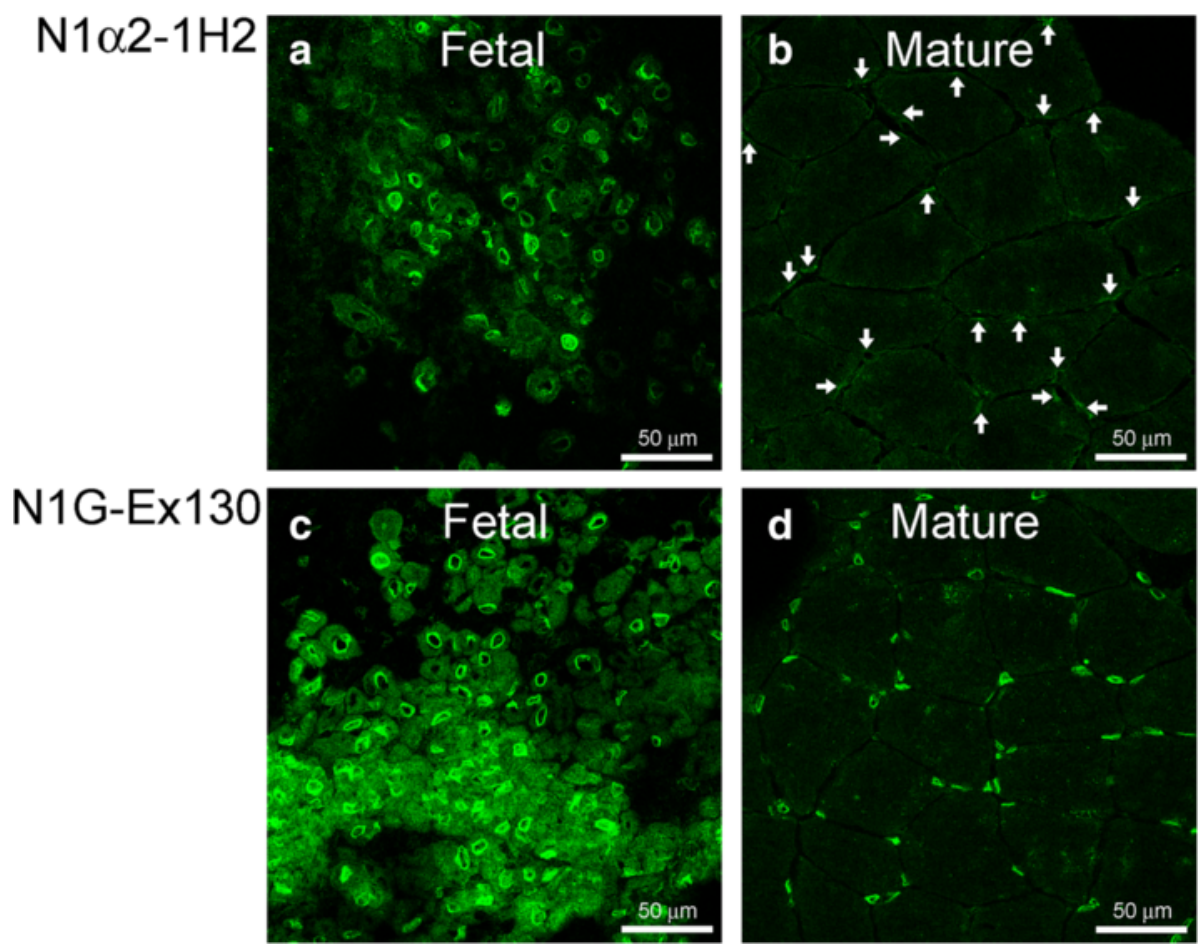

Fig. 6 Immunofluorescent staining of fetal and mature human muscle for nesprin-1-a2 and nesprin-1-giant. a mAb N1alpha2-1H2 stained nucle at the nuclear rim and gave less intense staining of cytoplasm in fetal muscle. b Nesprin-1-a2 was weakly stained in nuclei (white arrows) of mature muscle. Nesprin-1-a2 in mature muscle was much less obvious than that seen in fetal muscle. c Nesprin-1-giant (mAb N1G-Ex130) was found at the nuclear rim, with low level cytoplasmic stain, in fetal muscle. Nesprin-1-giant was also seen at the nuclear rim in adult skeletal muscle (d). Figure shows representative images of total of 3 fetal donors and 4 mature donors 


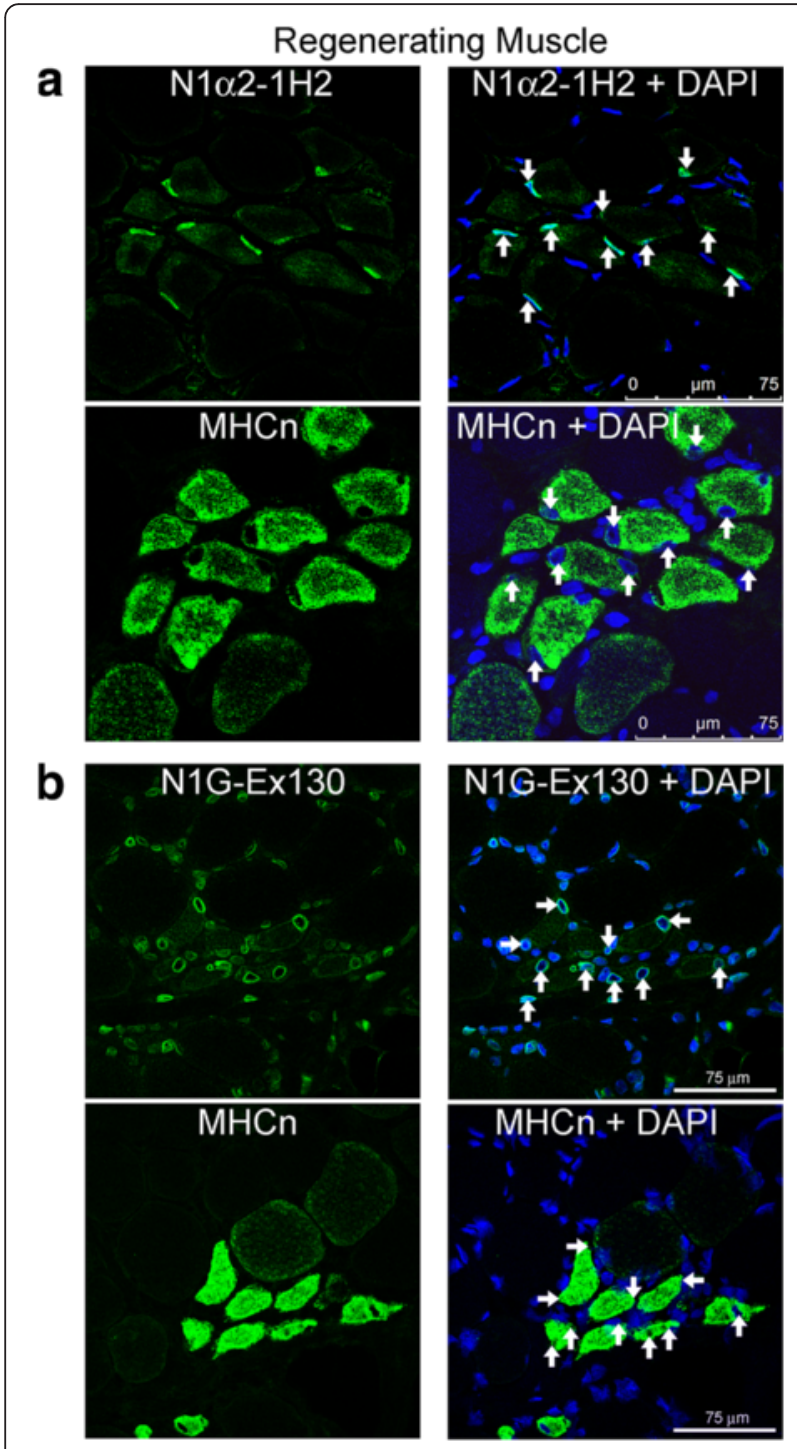

Fig. 7 Immunofluorescent staining for nesprin-1 in human skeletal muscle sections with regenerating muscle fibres. Individual microscopic fields are shown as paired images. The left image of each pair shows antibody stain (green) and the right image of each pair shows the merged antibody (green) and DAPI (blue) nuclear stain. a Serial sections from a patient with Duchenne muscular dystrophy, stained with mAb N1alpha2-1H2 (upper frame) and neonatal myosin ( $\mathrm{MHCn}$; regenerating fibres; lower frame). Staining for nesprin-1-a2 was most intense at the nuclear rim, but also faintly in the cytoplasm, in those fibres that were regenerating. $\mathbf{b}$ Serial sections from the same donor, stained for nesprin-1-giant (mAb N1G-Ex130; upper frame) and neonatal myosin ( $\mathrm{MHCn}$; lower frame) indicated that nesprin-1-giant was detected in all nuclei, but staining for nesprin-1giant at the nuclear rim was more intense, with faint cytoplasmic staining, in regenerating fibres. White arrows indicate the nuclei of regenerating fibres in the paired serial section

known [6, 28], including CPG2 (109kD) [29], GSRP-56 (56 kD) [30] and Drop1 [31]. More recently, Rajgor et al, $[10,27]$ have shown numerous isoforms that cover much of the $\mathrm{N}$-terminal half of the molecule, although their abundance and tissue distribution has not been precisely established. For this reason, we have extended the repertoire of available antibodies by producing a new $\mathrm{mAb}$, N1G-Ex130, which recognises only giant and beta isoforms of nesprin-1, plus a panel of new mAbs that recognises nesprin-1-giant and NOT beta isoforms (though they would also recognize some N-terminal KASH-less isoforms, if they were present).

In addition to $\mathrm{N}$-terminal isoforms lacking large $\mathrm{C}$ terminal regions, there are also known isoforms that lose only a short KASH sequence by alternative splicing (e.g. the last 99 amino-acids; [32]) and are otherwise similar to the known KASH+ isoforms. There are currently no antibodies that distinguish these KASH-less forms from their $\mathrm{KASH}+$ equivalents. Such KASH-less forms may have a putative nuclear localization signal (over 800 amino-acids from the nesprin-1 C-terminus [2]) and the KASH-less form of nesprin-1-giant in cerebellum has been shown to locate to the cytoplasm [32]. In embryonic stem cells, however, KASH-less forms of the related protein, nesprin-2, located to the nucleoplasm [22].

Using the new $\mathrm{mAb}$ tools described here for the first time, we have shown that nesprin-1- $\alpha 2$, the principal short form of nesprin-1 in cardiac and skeletal muscle $[1,22]$, appears after myoblast differentiation into early myotubes, remains at high levels in immature muscle fibres and becomes very weakly present by immunolocalization in most mature, adult muscle fibres (Figs. 6 \& 7). Nesprin-1-giant, the main product of the SYNE1 gene in most, if not all, tissues, was present at all stages of muscle development (Figs. 4 and 6), though its levels were also somewhat elevated in immature fibres relative to mature fibres (Fig. 7b). We have previously reported a decrease in nesprin-1 and an increase in nesprin-2 during the transition from immature to mature muscle fibres [26].

The clonal myoblast cell lines used in this study are a valuable resource because, unlike primary myoblast cultures, they do not contain variable proportions of non-muscle cells, such as fibroblasts. Immortalization does not seem to have affected their differentiation potential, we did not observe any effect of donor age on nesprin mRNA expression (Table 1) and the mRNA data from qPCR were consistent with the protein data from antibody studies (Fig. 4).

Fetal and adult muscle RNA samples, however, whether obtained commercially as extracted total RNA or locally as tissue stored for RNA extraction, gave very variable results in qPCR (Table 4). It seems likely that variability between samples, both in their "non-muscle" tissue content and in the storage and extraction conditions, might affect the levels of some mRNA species. Indeed, Table 4 suggests that low abundance mRNAs, like those for nesprins, give less consistent qPCR results than abundant species, like creatine kinase and 
Table 4 Relative expression of mRNA of nesprin-1 isoforms and markers of differentiation in normal human skeletal muscle

\begin{tabular}{|c|c|c|c|c|c|c|c|c|}
\hline & \multicolumn{3}{|c|}{ Fetal Skeletal Muscle } & \multicolumn{5}{|c|}{ Adult Skeletal Muscle } \\
\hline & Fetal 1 & Fetal 2 & Fetal 3 & Adult 1 (30y) & Adult 2 (18y) & Adult 3 (67y) & Adult 4 (85y) & Adult 5 \\
\hline N1-Giant & $269.0 \pm 127.7$ (8) & $290.3 \pm 36.6(4)$ & $5.3 \pm 3.3(5)$ & $172.4 \pm 27(5)$ & $113.6 \pm 16.5(4)$ & $119.2 \pm 30.4(6)$ & $47.5 \pm 9.3(5)$ & $86.8 \pm 25.9(3)$ \\
\hline$N 1-\beta 1$ & $2.4 \pm 1.9(5)$ & $1(2)$ & $<1(2)$ & $21.3 \pm 2.9(4)$ & $<1(2)$ & $<1(2)$ & $8.2 \pm 3.0(2)$ & $9.8 \pm 2.6(3)$ \\
\hline$N 1-\beta 2$ & $8.0 \pm 1.9(4)$ & $124.4 \pm 43.2(8)$ & $<1(3)$ & $4.9 \pm 1.7(4)$ & $<1(2)$ & $1(2)$ & $<1(3)$ & $1(2)$ \\
\hline $\mathrm{N} 1-\mathrm{a} 1$ & nd (1) & nd (1) & nd (1) & nd (1) & nd (1) & nd (1) & nd (1) & nd (1) \\
\hline$N 1-a 2$ & $120.8 \pm 33.9(4)$ & $66.2 \pm 18.1(5)$ & $10.3 \pm 2.3(5)$ & $17.8 \pm 5.8(4)$ & $48.6 \pm 12.7(6)$ & $140.3 \pm 25.4(5)$ & $30.1 \pm 5.3(5)$ & $55.4 \pm 18.2$ \\
\hline CKM & $4237 \pm 95(4)$ & $1611 \pm 295$ & $3404 \pm 971(5)$ & $33117 \pm 2320$ & $89214 \pm 10651$ & $117928 \pm 25917(4)$ & $20372 \pm 1290$ & $19033 \pm 1851$ \\
\hline Desmin & $11928 \pm 802(3)$ & $3260 \pm 1034(6)$ & $14542 \pm 3431$ & $38904 \pm 1567$ (3) & $84550 \pm 20608$ (4) & $82022 \pm 23108$ (5) & $37307 \pm 617$ (5) & $27197 \pm 4613$ \\
\hline
\end{tabular}


desmin mRNAs. However, the mRNA for nesprin-1- $\alpha 2$ was detected by qPCR in adult muscle (Table 4) in amounts, relative to nesprin-1-giant, consistent with the $111 \mathrm{kD}$ band in western blot (Fig. 3b and c). In other words, the mRNA was certainly present in adult muscle, although nesprin-1- $\alpha 2$ protein was detected weakly by immunofluorescence microscopy in normal adult muscle. One possibility is that the epitope recognized by our $\mathrm{mAb}$ at the $\mathrm{N}$-terminus of nesprin-1- $\alpha 2$ becomes masked in mature fibres in situ; it is not possible to test this hypothesis using different nesprin-1- $\alpha 2$-specific mAbs because the unique $\mathrm{N}$-terminal immunogen sequence for this isoform is very short. This seems the simplest way to reconcile $\mathrm{qPCR}$, western blot and immunofluorescence data at the present time.

A less likely possibility is that not all of the western blot band at around $111 \mathrm{kD}$ detected by mAb MANNES1E (Fig. 3b and c) is actually nesprin-1- $\alpha 2$; in a previous study, we showed that some prominent protein bands on western blots of skeletal muscle extracts, previously interpreted as known isoforms, were likely degradation products of the giant isoforms, since the corresponding mRNAs were barely detectable by qPCR [22]. It is also possible, but very unlikely, that larger muscle samples used for RNA extraction contain nesprin-1- $\alpha 2$-positive cells that are not seen in our small biopsy samples for immunofluorescence microscopy.

Missense mutations in the 3'-region of SYNE1 usually result in autosomal-dominant Emery-Dreifuss muscular dystrophy or inherited cardiomyopathy, with nesprin-1 function impaired, though perhaps not abolished [11-14]. The homozygous SYNE1 early termination mutation produces a severe, congenital muscular dystrophy phenotype, affecting several tissues [23], probably because expression of $\mathrm{KASH}+$ nesprin-1 is massively reduced. If the expression of nesprin-1- $\alpha 2$ had been unaffected, as predicted from the position of the early stop codon, it might have thrown light on roles for nesprin-1-giant that cannot be replaced by the short isoform, nesprin-1- $\alpha 2$. However, both the mRNA and protein levels of nesprin-1$\alpha 2$ were as much affected as was nesprin-1-giant by the mutation, although there does not appear to be any simple explanation for the reduced levels of nesprin-1- $\alpha 2$ mRNA. It is interesting that the mutation did not seem to affect in vitro myogenesis, measured by either myotube formation (Fig. 4b) or creatine kinase mRNA expression (Table 2). Nuclear re-positioning to the fibre periphery, however, does not occur until later stages of muscle development.

The detection of cytoplasmic nesprin-1 isoforms (both giant and alpha-2) in fetal muscle fibres, and to a lesser extent in myotubes, in addition to their principal location at the nuclear membrane, is not altogether surprising, since non-nuclear rim localizations in some circumstances have been reported previously $[22,26,33]$. The possibility that these are $\mathrm{KASH}$-less splice variants, unable to attach to the nuclear membrane, cannot be excluded.

Although we have no evidence that nesprin-1- $\alpha 2$ is involved in movement of nuclei during muscle formation, there is circumstantial evidence to suggest that this is one possibility. Nuclear positioning is disrupted in nesprin-1 knockout mice [34, 35] and nesprin-1-giant is known to link the nuclear rim to the actin cytoskeleton $[2,36]$. Although nesprin-1- $\alpha 2$ is unable to bind to actin via $\mathrm{CH}$ domains, it does share with the giant form, in its highly-conserved STAR domain [28], a LEWD motif that appears to be involved in binding kinesins [37]. Kinesins act as a linker to the microtubule system, which is involved in active movement of nuclei [38], including the movement of myonuclei along the longitudinal axis of the developing myotube [39-41]. This LEWD motif is the only part of the STAR domain shared by nesprin-4, a $44 \mathrm{kD}$ KASH-domain protein also known to bind microtubules via kinesins, but with a distribution that may be limited to tissues with secretory epithelial cells [19]. KASH5 lacks a LEWD motif and appears to bind to microtubules via dynein; like nesprin-4, it also exhibits a limited range of expression in spermatocytes, bone marrow and fetal liver [21]. It is possible that KASH proteins in the size range of $44-111 \mathrm{kD}$, such as nesprin-1- $\alpha 2$, carry out similar functions related to nuclear movements, but in specific tissues or at specific stages of development.

\section{Conclusions}

Expression of mRNA and protein of nesprin-1- $\alpha 2$, the skeletal and cardiac muscle-specific isoform of nesprin1 , is switched on during myogenesis, mirroring the expression of muscle-creatine kinase. The dynamic profile of nesprin-1- $\alpha 2$ expression suggests that it may be involved in some process occurring during early myofibre formation, such as re-positioning of nuclei. If the weak staining of nesprin-1- $\alpha 2$ in mature fibres is due to epitope masking, we might speculate that nesprin-1- $\alpha 2$ is carrying out one function in immature fibres, when it is accessible to antibody, and a different function in mature fibres, when antibody access is restricted.

\section{Methods}

\section{Muscle cell culture}

Clonal immortalized myoblast cell lines (Table 5) were from five human control donors without neuromuscular disease (aged 5 days and 25, 41, 53 and 79 years), from a congenital muscular dystrophy patient with a homozygous premature stop mutation in the nesprin-1 gene (Nucleotide: 23560 gaa to taa; Protein: E7854X; [23] and 
Table 5 Immortalized human myoblasts

\begin{tabular}{lll}
\hline Name & Donor & Donor Muscle \\
\hline C5d & Newborn (5.5 day), female & Quadriceps \\
C41yr & 25 years, male & Semitendinosus \\
C53yr & 41 years, male & Pectoralis Major \\
C79yr & 53 years, male & Quadriceps \\
Syne-1 E7854X & 79 years, female & Quadriceps \\
LMNA L380S & 16 years. SYNE1 c.23560 G > T, p.Glu7854* Homozygous & Paravertebral \\
LMNA del.K32 & 12 years, male. LMNA c.1139T > C, p.Leu380Ser Heterozygous & Paravertebral \\
LMNA R249W & 5 years, female. LMNA c.94_69delAAG, p.Lys32del Heterozygous & Gastrocnemius \\
\hline
\end{tabular}

Control, Syne-1 mutant [23] and LMNA mutant myoblasts [24, 25], immortalised as described [42]

from three congenital muscular dystrophy patients with mutations in the lamin A/C gene (Protein: L380S, del.K32 and R249W; [24, 25]. They were immortalized by transduction with human telomerase reverse transcriptase (hTERT) and cyclin-dependent kinase-4 (Cdk4) containing retroviral vectors, at the Institut de Myologie, Paris, as described previously [42]. They were cultured in skeletal muscle cell growth medium (Cat No: C-23060; PromoCell GmbH, Heidelberg, Germany) containing supplement mix (Cat No: C-39365; PromoCell) with $20 \%$ Fetal Bovine Serum (Cat No: 10270; Gibco; ThermoFisher Scientific, Paisley, UK). Differentiation was induced at $80 \%$ confluency by washing the adherent myoblasts in medium lacking serum and then culturing in DMEM (Cat No: 31966-021; Gibco; ThermoFisher Scientific) supplemented with Insulin (1721nM), Transferrin (68.7nM), Selenium (38.7nM) (ITS-X; Cat No: 51500-056; Gibco; ThermoFisher Scientific) and Penicillin-Streptomycin (Cat No: DE17-603E; Lonza; Verviers, Belgium). After a further 4 days of cell culture, over $80 \%$ of the cells had fused into myotubes.

\section{RT-PCR and qPCR}

Total RNA was prepared from cultured cells and from skeletal muscle samples using RNeasy Plus Mini Kit (Qiagen) and quantified with a NanoDrop ND1000 spectrophotometer. Human skeletal muscle total RNA were obtained from the following sources: Fetal 1 (19 week female fetus; cat no: T5595-7587; lot no: L14020669) and Adult 1 (30 year female; cat no: T5595-7379; lot no: L11033012) were both purchased from United States Biological (Swampscott, MA 01907). Fetal 3 (18 week female fetus; cat no: 540181; lot no: 0006260887) and Adult 4 (85 year female; cat no: 540029; lot no: 0006167155) were both purchased from Agilent Technologies (Stockport, Cheshire, SK8 3GR, UK). Adult 5 from the First Choice Human Total RNA Survey Panel (cat no: AM6000; lot no: 1004067; [22]) was purchased from Ambion Inc (Austin, TX 78744). Fetal 2 muscle was obtained from the MRC Centre for Neuromuscular Disease Biobank, London. Adult 2 muscle (18 year male) and Adult 3 muscle (67 year female) were obtained during routine surgery at RJAH Orthopaedic Hospital, Oswestry. Total RNA (maximum of $2.5 \mu \mathrm{g}$ in a $20 \mu \mathrm{L}$ reaction) was reverse transcribed (SuperScript VILO cDNA Synthesis Kit; Applied Biosystems) and then diluted with sterile water, in order to achieve the cDNA equivalent of 10ng total RNA for each $20 \mu \mathrm{L}$ reaction in the qPCR plate.

Forward primers for the short isoforms of nesprin-1 were designed to recognise unique sequences in the 5' UTR of each isoform [22]. Primer sequences for M-creatine kinase (accession: NM_001824) were F: GCTCGTCCGAAGTAGAACAGGTG and R: GGTTG GAACTCTGGTTGAAACTG (282 bp product size) and for desmin (accession: NM_001927) were F: GC TCAACGTGAAGATGGCCCT and R: CTGCTGCTG TGTGGCCTCACT (223 bp product size). Primer pairs were tested by conventional PCR and products were confirmed by DNA sequencing [22]. Primer pairs were chosen which gave dissociation curves with single peaks.

Relative quantitative PCR was performed with an ABI 7500 Real Time PCR system (Applied Biosystems) using SYBR green detection, as previously described [22]. For each preparation of cDNA, two endogenous controls (Beta-actin and GAPDH) were amplified along with each target sequence. The $2^{-\Delta C T}$ method $[43,44]$ was used to calculate the quantity of target transcripts relative to the two endogenous reference transcripts. The $C_{T}$ (cycle threshold) value was plotted against the $\log$ cDNA dilution in order to calculate the efficiency of the primer pairs [44].

\section{Hybridoma production Peptide immunogens}

The peptide VVAEDLSALRMAEDGC, with Keyhole Limpet Hemocyanin (KLH) conjugated to its C-terminus 
(Davids Biotechnologie GmbH, Regensburg, Germany), was used to immunize $\mathrm{CD} 1$ mice for production of $\mathrm{mAbs}$ against the unique $\mathrm{N}$-terminal sequence of Nesprin-1- $\alpha 2$. The peptide SKASEIEYKLGKVNDRC, with KLH conjugated to its C-terminus (AltaBioscience, Birmingham UK), was used to immunize Balb/C mice for production of mAbs against a sequence within exon 130 of Nesprin-1giant. For hybridoma production (described in detail previously: Nguyen thi Man and Morris, 2010 [45]), spleen cells from immunized mice were fused with mouse myeloma cell line Sp2/O and 960 wells were screened by ELISA against unconjugated peptides. ELISA-positives were screened by immunofluorescence microscopy on unfixed human muscle sections and western blotting with human skeletal muscle extracts (for exon $130 \mathrm{mAbs)}$ ), before cloning by limiting dilution.

\section{Recombinant fusion protein immunogen}

Nesprin-1-giant cDNA (corresponding to amino acids 5209-5570) in pGEX vector was used to transform $E$. coli BL21(DE3) which were induced by IPTG to give a GSTfusion protein. After incubation the cells were washed with TNE buffer and sonicated sequentially with TNE, 2M urea, $4 \mathrm{M}$ urea, $6 \mathrm{M}$ urea and $8 \mathrm{M}$ urea. Fusion protein in the $6 \mathrm{M}$ urea fraction was used in the protocol for immunization of $\mathrm{Balb} / \mathrm{C}$ mice for production of mAbs [45]. ELISA plates were coated with either the GST-nesprin-1 amino acids 5209-5570 fusion protein used for immunization or with an unrelated GST-fusion protein (GST-MSH3), in order to eliminate those mAbs reacting with the GST fusion tag. For further refinement of the epitope positions, ELISAs were also performed with plates coated with recombinant protein GST-nesprin-1-giant amino acids 5377-5570 (nesprin-1 isoform p23, Accession No: JQ754364), in order to identify those mAbs with epitopes at the $\mathrm{C}$-terminal end of the original immunogen. ELISA-positives were screened by immunofluorescence microscopy and western blotting, before cloning by limiting dilution. Further epitope mapping was performed by testing the mAbs for immunolocalization with COS-7 cells transfected with $\mathrm{pCMV} /$ nesprin-1-beta-1 (amino acids 1-97). These mAbs were therefore classed as reacting with nesprin-1-giant exons 81-84 (amino acids 5209-5376) or nesprin-1-giant exons 84-86 (amino acids 5377-5476), which does not include any sequence from nesprin-1-beta-1.

Other mAbs used in this study were: MANNES1A (for immunofluorescence microscopy) and MANNES1E (for western blot), both of which recognise the C-terminal region of nesprin-1 [26].

\section{SDS-polyacrylamide gel electrophoresis and Western blotting}

Cell lysis buffer (125 mM Tris pH 6.8, 2 \% SDS, 5 \% 2beta mercaptoethanol, $5 \%$ glycerol with protease inhibitors: Sigma P8340 and 1mM PMSF) was used for the extraction of cultured cells and tissue lysis buffer (50mM Tris pH 6.8, 1 \% EDTA, $10 \%$ SDS, $5 \%$ beta mercaptoethanol, $10 \%$ glycerol with protease inhibitors) was used for the extraction of tissue samples $(250 \mathrm{mg} / \mathrm{ml})$. Bromophenol blue was added to the samples which were then boiled and separated by SDS-PAGE using 4 to $12 \%$ polyacrylamide gels (Ref: NW04125BOX; ThermoFisher Scientific) and then electroblotted onto nitrocellulose membranes (Protan BA85, Whatman). Non-specific sites were blocked with $5 \%$ skimmed milk protein and the membranes then incubated with monoclonal antibody supernatants (diluted: 1/10, except MANNES1E: $1 / 50$ and MANDRA1: 1/100), followed by washing and incubation with secondary antibody (peroxidase-labelled rabbit anti-mouse immunoglobulins; 1/1000, Dako, Denmark). Antibody reacting bands were detected with SuperSignal West Femto chemiluminescent reagent (Cat No: 34094; ThermoFisher Scientific) and visualized with a ChemiDoc Touch imaging system (BioRad Ltd.).

\section{Immunofluorescence microscopy}

Immunohistochemistry was performed on cultured cells on coverslips that had been fixed with $50 \%$ acetone, $50 \%$ methanol and then washed with PBS and also performed on unfixed cryostat sections of human muscle. Monoclonal antibodies in culture supernatants were diluted and incubated on specimens for $1 \mathrm{~h}$. N1alpha2-1H2 was diluted 1:1 to $1: 3$ in PBS and all other nesprin-1 mAbs were diluted 1:3 in PBS. Following incubation with the monoclonal antibody, specimens were washed with PBS and then incubated with goat antimouse ALEXA 488 (Molecular Probes, Eugene, Oregon, USA) secondary antibody (diluted to $5 \mu \mathrm{g} / \mathrm{ml}$ in PBS containing $1 \%$ horse serum, $1 \%$ fetal bovine serum, $0.1 \% \mathrm{BSA}$ ). After $50 \mathrm{~min}$, DAPI (diamidino phenylindole at $200 \mathrm{ng} / \mathrm{ml}$ ) was also added to specimens to counterstain nuclei. After incubation for 10 min with DAPI, specimens were washed with PBS and mounted in Hydromount (Merck). Images were acquired by sequential scanning with a Leica TCS SP5 spectral confocal microscope (Leica Microsystems, Milton Keynes, UK). For peptide competition experiments, mAb N1alpha2-1H2 was pre-incubated with $1 \mathrm{mg} / \mathrm{ml}$ unconjugated peptide (VVAEDLSALRMAEDGC) or unrelated control peptide (IFSHQQVKKLKETFAFIQQLC) for $1 \mathrm{~h}$ at room temperature.

\section{Abbreviations}

$\mathrm{CH}$, Calponin homology; CKM, Creatine kinase, muscle; EDMD, Emery-Dreifuss muscular dystrophy; KASH, Klarsicht, ANC-1 and Syne homology; KLH, Keyhole limpet hemocyanin; LINC, Linker of Nucleoskeleton and Cytoskeleton; SUN, Sad-1, UNC-84 


\section{Acknowledgments}

The Platform for Immortalization of Human Cells, Institute of Myology, Paris, for the immortalization of human myoblasts.

\section{Funding}

This study was supported by British Heart Foundation grant (PG/11/71/29091) to IH and GEM; RJAH Orthopaedic Hospital Institute of Orthopaedics (RPG 149) to IH and GEM; The Vietnam National Foundation for Science and Technology Development (NAFOSTED) (106.06-2010.62) to NTD; Muscular Dystrophy Association Research Infrastructure grant (173057) to GEM; Association Française contre les Myopathies to KM.

\section{Availability of data and materials}

Monoclonal antibodies are available from the MDA monoclonal antibody resource, Oswestry, UK (http://www.glennmorris.org.uk/mabs/WCIND.htm), which is supported by the Muscular Dystrophy Association, USA.

\section{Authors' contributions}

Conceived and designed the experiments: IH, NTD, QZ, CMS, GEM; Hybridoma production: IH, GEM; Quantitative PCR: IH, NTD, LTL; Immunofluorescence microscopy: IH, LTL, CAS; Myoblast immortalization: KM; Analysed the data: IH, NTD, QZ, LTL, CAS, CMS, GEM; Wrote the manuscript: IH, QZ, CMS, GEM. Revised the manuscript: IH, GEM. All authors read and approved the final manuscript.

\section{Competing interests}

The authors declare that they have no competing interests.

\section{Consent for publication}

Written informed consent to publish personal data was obtained from adult donors and from the parents or legal guardians of donors under 16 years.

\section{Ethical approval and consent to participate}

This study has been approved by the Robert Jones and Agnes Hunt Orthopaedic Hospital Research Committee. Muscle biopsies for isolation of myoblasts were obtained following written informed consent in accordance with European recommendations and French legislation. Human muscle biopsies were obtained following written informed consent using protocols approved by the MRC Centre for Neuromuscular Disease Biobank, London and the Robert Jones and Agnes Hunt Orthopaedic Hospital. Written parental or legal guardian informed consent was obtained for all participants under the age of 16 years. The monoclonal antibody production protocol was approved by the Keele University Animal Welfare and Ethical Review Body.

\section{Author details}

'Wolfson Centre for Inherited Neuromuscular Disease, RJAH Orthopaedic Hospital, Oswestry, SY10 7AG, UK. ${ }^{2}$ Institute for Science and Technology in Medicine, Keele University, Keele ST5 5BG, UK. Institute of Genome Research (IGR), Vietnam Academy of Science and Technology (VAST), Hanoi, Vietnam. ${ }^{4}$ Cardiovascular Division, James Black Centre, King's College, London SE5 9NU, UK. ${ }^{5}$ Dubowitz Neuromuscular Centre, Institute for Child Health and Great Ormond Street Hospital, London WC1 1EH, UK. ${ }^{6}$ Sorbonne Universités, UPMC Univ Paris 06, INSERM UMRS974, CNRS FRE3617, Center for Research in Myology, 47 Boulevard de l'hôpital, 75013 Paris, France.

\section{Received: 7 April 2016 Accepted: 20 June 2016}

\section{Published online: 27 June 2016}

\section{References}

1. Apel ED, Lewis RM, Grady RM, Sanesi JR. Syne-1, a dystrophin- and klarsicht-related protein associated with synaptic nuclei at the neuromuscular junction. J Biol Chem. 2000;275:31986-95.

2. Zhang Q, Skepper JN, Yang F, Davies JD, Hegyi L, Roberts RG, et al. Nesprins: a novel family of spectrin-repeat-containing proteins that localize to the nuclear membrane in multiple tissues. J Cell Sci. 2011;114:4485-98.

3. Zhen YY, Libotte T, Munck M, Noegel AA, Korenbaum E. NUANCE, a giant protein connecting the nucleus and actin cytoskeleton. J Cell Sci. 2002;115: 3207-22.

4. Zhang Q, Ragnauth CD, Skepper JN, Worth NF, Warren DT, Roberts RG, et al. Nesprin-2 is a multi-isomeric protein that binds lamin and emerin at the nuclear envelope and forms a subcellular network in skeletal muscle. J Cell Sci. 2005;118:673-87.
5. Mislow JM, Kim MS, Davis DB, McNally EM. Myne-1, a spectrin repeat transmembrane protein of the myocyte inner nuclear membrane, interacts with lamin A/C. J Cell Sci. 2002;115:61-70.

6. Padmakumar VC, Abraham S, Braune S, Noegel AA, Tunggal B, Karakesisoglou I, Korenbaum E. Enaptin, a giant actin-binding protein, is an element of the nuclear membrane and the actin cytoskeleton. Exp Cell Res. 2004;295:330-9.

7. Sosa BA, Rothballer A, Kutay U, Schwartz TU. LINC complexes form by binding of three KASH peptides to domain interfaces of trimeric SUN proteins. Cell. 2012;149:1035-47.

8. Zhou Z, Du X, Cai Z, Song X, Zhang H, Mizuno T, et al. Structure of Sad1UNC84 homology (SUN) domain defines features of molecular bridge in nuclear envelope. J Biol Chem. 2012;287:5317-26.

9. Lombardi ML, Lammerding J. Keeping the LINC: the importance of nucleocytoskeletal coupling in intracellular force transmission and cellular function. Biochem Soc Trans. 2011;39:1729-34.

10. Rajgor D, Shanahan CM. Nesprins: from the nuclear envelope and beyond. Expert Rev Mol Med. 2013;15:e5.

11. Zhang Q, Bethmann C, Worth NF, Davies JD, Wasner C, Feuer A, et al. Nesprin-1 and -2 are involved in the pathogenesis of Emery Dreifuss muscular dystrophy and are critical for nuclear envelope integrity. Hum Mol Genet. 2007:16:2816-33.

12. Puckelwartz MJ, Kessler EJ, Kim G, Dewitt MM, Zhang Y, Earley JU, et al. Nesprin-1 mutations in human and murine cardiomyopathy. J Mol Cell Cardiol. 2010:48:600-8

13. Li C, Zhou B, Rao L, Shanahan CM, Zhang Q. Novel nesprin-1 mutations in human dilated cardiomyopathy. Nuclear envelope disease and chromatin organization meeting, Cambridge, 2011; Abstract P028.

14. Fanin M, Savarese M, Nascimbeni AC, Di Fruscio G, Pastorello E, Tasca E, et al. Dominant muscular dystrophy with a novel SYNE1 gene mutation. Muscle Nerve. 2015:51:145-7.

15. Bione S, Maestrini E, Rivella S, Mancini M, Regis S, Romeo G, Toniolo D. Identification of a novel X-linked gene responsible for Emery-Dreifuss muscular dystrophy. Nat Genet. 1994;8:323-7.

16. Bonne G, Di Barletta MR, Varnous S, Bécane HM, Hammouda EH, Merlini L, et al. Mutations in the gene encoding lamin A/C cause autosomal dominant Emery-Dreifuss muscular dystrophy. Nat Genet. 1999:21:285-8.

17. Meinke P, Nguyen TD, Wehnert MS. The LINC complex and human disease. Biochem Soc Trans. 2011:39:1693-7.

18. Wilhelmsen $\mathrm{K}$, Litjens $\mathrm{SH}$, Kuikman I, Tshimbalanga $\mathrm{N}$, Janssen $\mathrm{H}$, van den Bout l, et al. Nesprin-3, a novel outer nuclear membrane protein, associates with the cytoskeletal linker protein plectin. J Cell Biol. 2005;171:799-810.

19. Roux KJ, Crisp ML, Liu Q, Kim D, Kozlov S, Stewart CL, Burke B. Nesprin 4 is an outer nuclear membrane protein that can induce kinesin-mediated cell polarization. Proc Natl Acad Sci U S A. 2009;106:2194-9.

20. Morimoto A, Shibuya H, Zhu X, Kim J, Ishiguro K, Han M, Watanabe Y. A conserved KASH domain protein associates with telomeres, SUN1, and dynactin during mammalian meiosis. J Cell Biol. 2012;198:165-72.

21. Horn HF, Kim DI, Wright GD, Wong ES, Stewart CL, Burke B, Roux KJ. A mammalian KASH domain protein coupling meiotic chromosomes to the cytoskeleton. J Cell Biol. 2013;202:1023-39.

22. Duong NT, Morris GE, Lam le T, Zhang Q, Sewry CA, Shanahan CM, Holt I. Nesprins: tissue-specific expression of epsilon and other short isoforms. PLoS One. 2014:9:e94380

23. Voit T, Cirak S, Abraham S, Karakesisoglou I, Parano E, Pavone P, et al. Congenital muscular dystrophy with adducted thumbs, mental retardation, cerebellar hypoplasia and cataracts is caused by mutation of Enaptin (Nesprin-1): The third nuclear envelopathy with muscular dystrophy (Abstract C.O.4). Neuromuscul Disord. 2007;17:833-834.24.

24. Quijano-Roy S, Mbieleu B, Bönnemann CG, Jeannet PY, Colomer J, Clarke $\mathrm{NF}$, et al. De novo LMNA mutations cause a new form of congenital muscular dystrophy. Ann Neurol. 2008:64:177-86.

25. Bertrand AT, Ziaei S, Ehret C, Duchemin H, Mamchaoui K, Bigot A, et al. Cellular microenvironments reveal defective mechanosensing responses and elevated YAP signaling in LMNA-mutated muscle precursors. J Cell Sci. 2014;127:2873-84

26. Randles KN, Lam LT, Sewry CA, Puckelwartz M, Furling D, Wehnert M, et al. Nesprins, but not sun proteins, switch isoforms at the nuclear envelope during muscle development. Dev Dyn. 2010;239:998-1009.

27. Rajgor D, Mellad JA, Autore F, Zhang Q, Shanahan CM. Multiple novel nesprin-1 and nesprin-2 variants act as versatile tissue-specific intracellular scaffolds. PLoS One. 2012;7:e40098. 
28. Simpson JG, Roberts RG. Patterns of evolutionary conservation in the nesprin genes highlight probable functionally important protein domains and isoforms. Biochem Soc Trans. 2008;36:1359-67.

29. Cottrell JR, Borok E, Horvath TL, Nedivi E. CPG2: a brain- and synapsespecific protein that regulates the endocytosis of glutamate receptors. Neuron. 2004:44:677-90.

30. Kobayashi Y, Katanosaka Y, Iwata Y, Matsuoka M, Shigekawa M, Wakabayashi S. Identification and characterization of GSRP-56, a novel Golgi-localized spectrin repeat-containing protein. Exp Cell Res. 2006;312:3152-64.

31. Marmé A, Zimmermann HP, Moldenhauer G, Schorpp-Kistner M, Müller C, Keberlein $\mathrm{O}$, et al. Loss of Drop1 expression already at early tumor stages in a wide range of human carcinomas. Int J Cancer. 2008;123:2048-56.

32. Razafsky D, Hodzic D. A variant of Nesprin1 giant devoid of KASH domain underlies the molecular etiology of autosomal recessive cerebellar ataxia type I. Neurobiol Dis. 2015:78:57-67.

33. Rajgor D, Mellad JA, Soong D, Rattner JB, Fritzler MJ, Shanahan CM. Mammalian microtubule P-body dynamics are mediated by nesprin-1. J Cell Biol. 2014;205:457-75.

34. Zhang $X$, Xu R, Zhu B, Yang $X$, Ding $X$, Duan $S$, et al. Syne- 1 and Syne-2 play crucial roles in myonuclear anchorage and motor neuron innervation. Development. 2007;134:901-8.

35. Zhang J, Felder A, Liu Y, Guo LT, Lange S, Dalton ND, et al. Nesprin 1 is critical for nuclear positioning and anchorage. Hum Mol Genet. 2010;19: 329-41.

36. Zhang Q, Ragnauth C, Greener MJ, Shanahan CM, Roberts RG. The nesprins are giant actin-binding proteins, orthologous to Drosophila melanogaster muscle protein MSP-300. Genomics. 2002;80:473-81.

37. Wilson MH, Holzbaur EL. Nesprins anchor kinesin-1 motors to the nucleus to drive nuclear distribution in muscle cells. Development. 2015;142:218-28.

38. Fridolfsson HN, Starr DA. Kinesin-1 and dynein at the nuclear envelope mediate the bidirectional migrations of nuclei. J Cell Biol. 2010;191:115-28.

39. Metzger T, Gache V, Xu M, Cadot B, Folker ES, Richardson BE, et al. MAP and kinesin-dependent nuclear positioning is required for skeletal muscle function. Nature. 2012;484:120-4.

40. Wilson MH, Holzbaur ELF. Opposing microtubule motors drive robust nuclear dynamics in developing muscle cells. J Cell Sci. 2012;125:4158-69.

41. Cadot B, Gache V, Vasyutina E, Falcone S, Birchmeier C, Gomes ER. Nuclear movement during myotube formation is microtubule and dynein dependent and is regulated by Cdc42, Par6 and Par3. EMBO Rep. 2012;13: 741-9.

42. Mamchaoui K, Trollet C, Bigot A, Negroni E, Chaouch S, Wolff A, et al. Immortalized pathological human myoblasts: towards a universal tool for the study of neuromuscular disorders. Skelet Muscle. 2011;1:34.

43. Livak KJ, Schmittgen TD. Analysis of relative gene expression data using real-time quantitative PCR and the 2(-Delta Delta C(T)) Method. Methods. 2001;25:402-8

44. Schmittgen TD, Livak KJ. Analyzing real-time PCR data by the comparative C(T) method. Nat Protoc. 2008:3:1101-8.

45. Nguyen TM, Morris GE. A rapid method for generating large numbers of high-affinity monoclonal antibodies from a single mouse. In: Walker JM, editor. The Protein Protocols Handbook. 3rd ed. Totowa NJ: Humana Press; 2010. p. 1961-74.

\section{Submit your next manuscript to BioMed Central and we will help you at every step:}

- We accept pre-submission inquiries

- Our selector tool helps you to find the most relevant journal

- We provide round the clock customer support

- Convenient online submission

- Thorough peer review

- Inclusion in PubMed and all major indexing services

- Maximum visibility for your research

Submit your manuscript at www.biomedcentral.com/submit
Biomed Central 